\title{
Pyrophosphate Enhancement of Thyrocalcitonin Effect in Tissue Culture
}

\author{
HAJIME ORIMO, TAKUO FUJITA AND MASAKI YOSHIKAWA \\ Department of Geriatrics, Faculty of Medicine, \\ University of Tokyo, Tokyo
}

Synopsis

\begin{abstract}
In vitro bone culture technique was developed by the modification of the Goldhaber's method. Using this method, pyrophosphate was found to inhibit PTH induced bone resorption as judged by the release of previously incorporated $\mathrm{Ca} 45$ into the medium and by the histological findings. Furthermore, addition of pyrophosphate markedly enhanced the thyrocalcitonin inhibition of bone resorption in this system. These findings, together with the previous findings that pyrophosphate enhances the hypocalcemic response to TC in vivo, strongly suggest the favorable effect of the combined use of pyrophosphate and TC in the bone diseases characterized by excessive bone resorption.
\end{abstract}

Thyrocalcitonin (TC) exerts its hypocalcemic effect through the inhibition of bone resorption. (Munson et al., 1966).

Direct evidence for such a mechanism was obtained in tissue culture by Friedman and Raisz (1965), Aliapoulios et al. (1966), Gaillard (1966) and Reynolds et al. (1968). The administration of phosphate has also been shown to lower blood calcium probably through increasing deposition of bone salt. (Pechet et al., 1967).

Furthermore, phosphate markedly enhanced the hypocalcemic response to $\mathrm{TC}$ in rats. (Hirsch 1968; Orimo et al., 1969).

In the previous paper, we have demonstrated the hypocalcemic effect of pyrophosphate and also its enhancing effect of TC in rats. (Orimo et al., 1969).

In the present report, we have studied the in vitro effect of pyrophosphate on bone resorption in tissue culture and also its enhancing effect of TC in this system.

Received for publication May 30, 1969.

\section{Materials and Methods}

The methods used were the modification of Goldhaber's technique. (Goldhaber, 1965). Pregnant swiss albino mice of the ICR strain were given s.c. injections of $30 \mu \mathrm{c}$ of $\mathrm{Ca}^{45}$ on the 16th day of gestation. Calvariae of 4 to 5 day old litter which were labelled with $\mathrm{Ca} 45$ are then aseptically removed and attached to a rectangular coverslip by covering the tissue with a thin film of 2 parts chicken plasma to 1 part chick embryo extract. After clotting, each coverslip was inserted into the well of a Leighton tube, and covered with $2 \mathrm{ml}$ of a supernatant fluid composed of $80 \%$ hearted horse serum, $10 \%$ Gey's salt solution containing $100 \mu$ each of penicillin and streptomycin. The tubes are gassed briefly with a mixture of $50 \% \mathrm{O}_{2}$ and $50 \% \mathrm{~N}_{2}$, stoppered, and placed horizontally in a rotor at $37^{\circ} \mathrm{C}$. The media are changed every other day unless otherwise specified and aliquuts counted for $\mathrm{Ca}^{45}$ activity by liquid scintillation counter. Measurement of the release of previously incorporated $\mathrm{Ca}^{45}$ was used as the parameter of quantitating bone resorption. (Raisz. 1965). After the termination of the experiments usually lasting for 7 days, bone containing coverslips are removed from the tubes, fixed overnight in $10 \%$ neutral formalin, and then stained by the Von Kossa method. The stained calvariae of each experimental group are mounted on separate microscopic slides, and the groups to be compared are photographed together $(4 \sim 5$ specimen per group). TC (27 MRC 
$\mu / \mathrm{mg}$ ), which was a gift of Eli Lilly and Company was dissolved in $0.1 M \mathrm{Na}$ acetate and pyrophosphate solution was prepared by dissolving $\mathrm{Na}_{4} \mathrm{P}_{2} \mathrm{O}_{7} \cdot 10 \mathrm{H}_{2} \mathrm{O}$. These materials are sterilized by passing through the millipore filter and the volume of $0.1 \mathrm{~m} l$ was added to the medium at the time when the medium was changed. The data in each experiment were subjected to analysis of variance. The standard errors were obtained from the residual error term of the analysis of variance. The means were compared using the multiple comparison test of Hartley. (Snedecor et al., 1967).

\section{Results}

\section{Effect of pyrophosphate on bone re- sorption}

As shown in Figure 1, addition of 5 and $10 \mu M$ of pyrophosphate significantly inhibited the increased release of $\mathrm{Ca}_{45}$ induced by PTE $48 \mathrm{hrs}$. after the incubation in a dose related manner. Gross appearance of the calvariae which were stained by Von Kossa method after a 6 day culture of another experiment of similar design also suggested the inhibition of PTE induced bone resorption by pyrophosphate. (Fig. 2) As shown in the next experiment, addition of graded doses of pyrophosphate demonstrated a dose related inhibition of $\mathrm{Ca}^{45}$ release in PTE stimulated cultures.

(Fig. 3) In these experiments, medium was changed every other day.

\section{Effect of graded dose of TC on bone resorption}

As shown in Figure 4, there was a doserelated inhibition of $\mathrm{Ca} 45$ release in. PTE stimulated culture by TC during $48 \mathrm{hrs}$. incubation. It was found that TC $15 \mathrm{MRC} \mathrm{mU} /$ tube caused the maximal inhibition of PTE stimulated $\mathrm{Ca}^{4} 5$ release during 48 hrs. (Fig. 4)

\section{Time course study with TC}

When TC was added to the medium initially, it resulted in the marked inhibition of $\mathrm{Ca}_{45}$ release in PTE treated medium during the first 2 days. Subsequently this inhibitory effect of

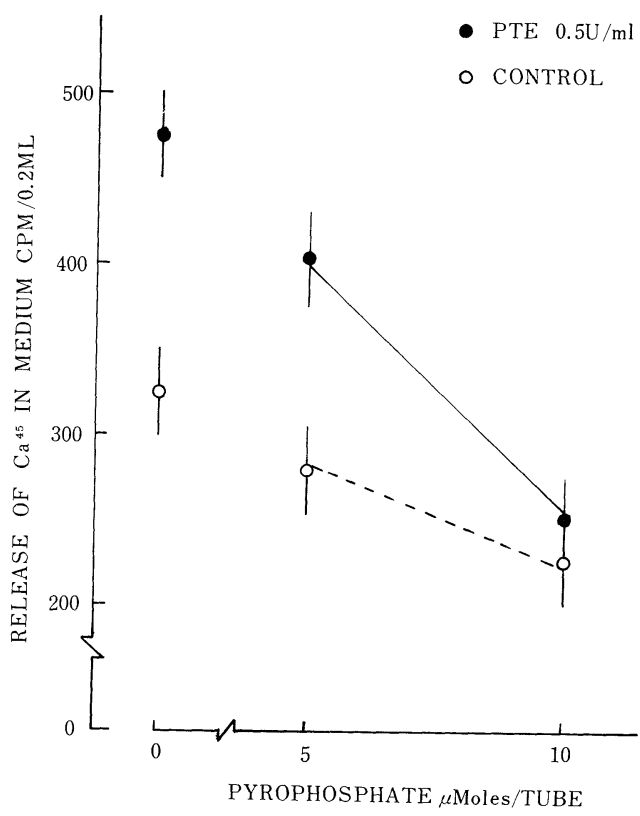

Fig. 1. Pyrophosphate inhibition of the release of previously incorporated $\mathrm{Ca}^{45}$ into the medium during $48 \mathrm{hrs}$. in control and in PTE stimulated cultures. Each point represents the mean of 5 cultures and the standard error is shown by the vertical line.

TC became less marked and after the continuation of the cultures for 4 days, $15 \mathrm{mU}$ of TC, which completely inhibited the PTE effect during the first 2 days, became no more effective on $4 \sim 6$ days. However, higher dose of TC was still effective at this time period, although the magnitude of the inhibition was greatly diminished as compared with that of the first 2 days of culture. (Fig. 5)

\section{Enhancement of TC effect by pyro- phosphate}

As shown in Figure 6, TC 5 mU significantly inhibited the increased release of $\mathrm{Ca}_{45}$ induced by PTE $48 \mathrm{hrs}$. after the incubation. Addition of pyrophosphate, $1 \mu$ moles at dose which was ineffective alone, markedly enhanced the inhibitory effect of TC.

Since the addition of pyrophosphate alone at this dose was ineffective, this might be 


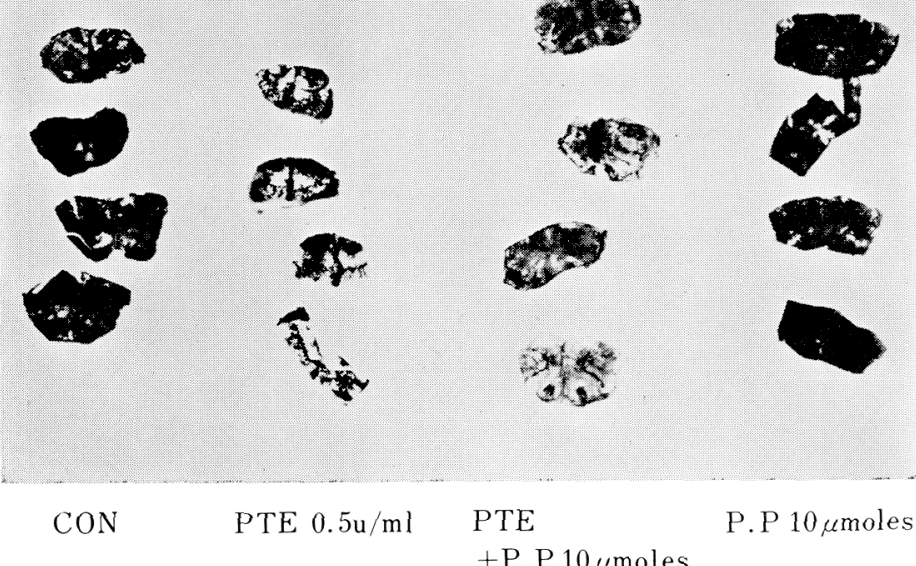

Fig. 2. Pyrophosphate inhibition of bone resorption induced by PTE in a 6 day culture

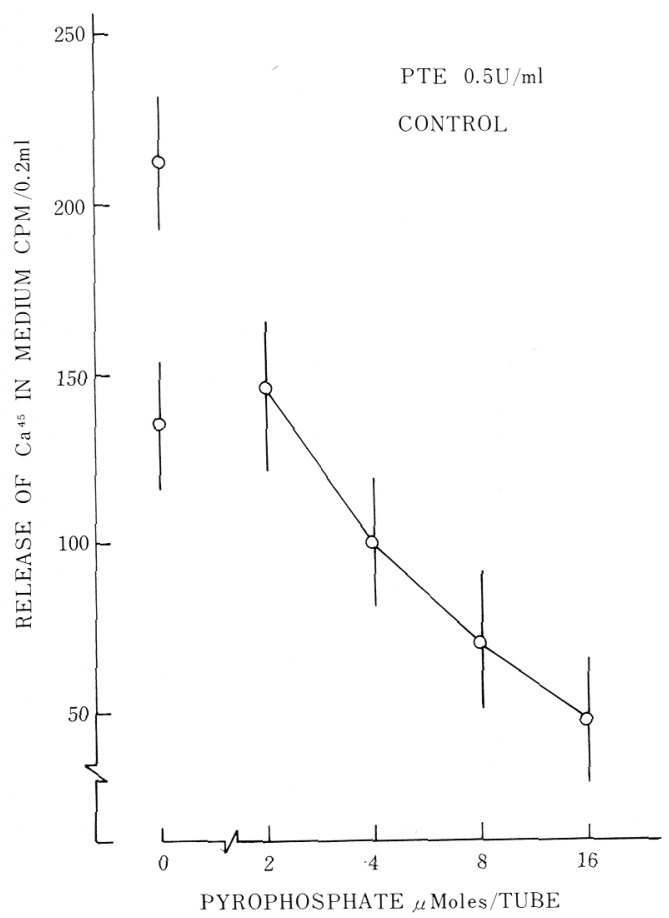

Fig. 3. Effect of increasing dose of pyrophosphate on the release of $\mathrm{Ca}^{45}$ in PTE stimulated cultures. Each point represents the mean of 5 cultures.

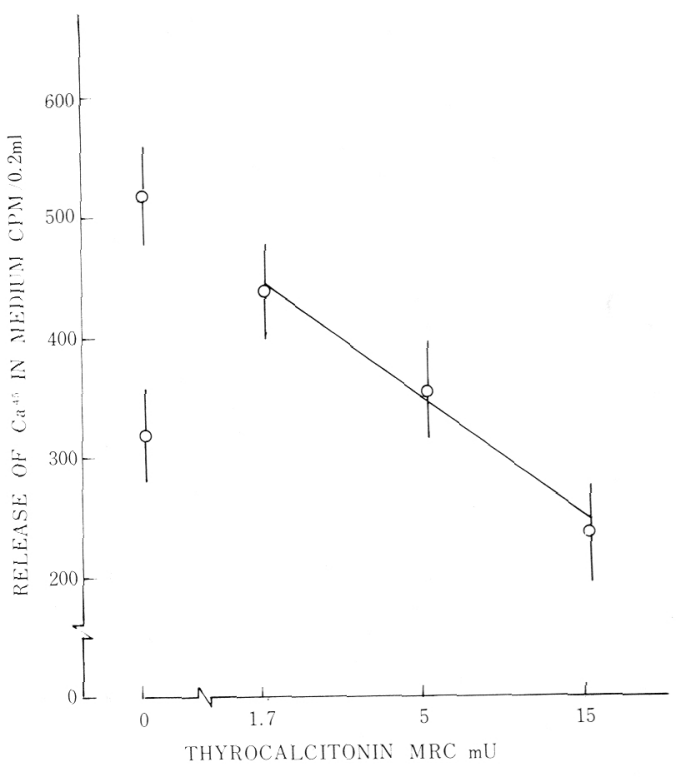

Fig.4. Thyrocalcitonin inhibition of the release of $\mathrm{Ca} 45$ in PTE stimulated cultures during $48 \mathrm{hrs}$. Each point represents the mean of 5 cultures. 


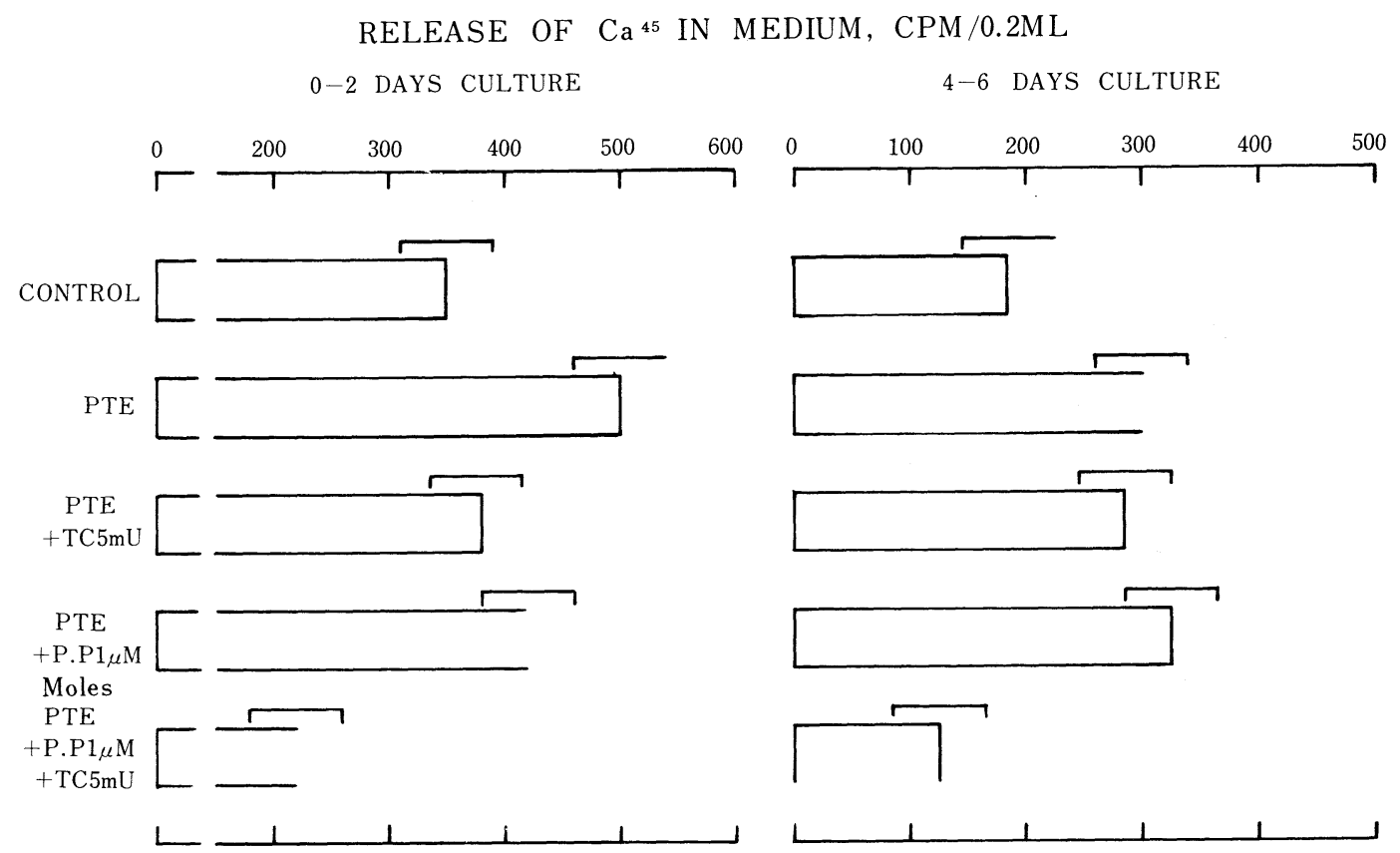

Fig. 5. Diminished inhibitory effect of $\mathrm{TC}$ on the release of $\mathrm{Ca} 45$ in prolonged PTE stimulated cultures Each point represents the mean of 5 cultures

RELEASE OF Ca ${ }^{45}$ IN MEDIUM, CPM/0.2ML

$$
\text { 0-2 DAYS CULTURE } \quad 4-6 \text { DAYS CULTURE }
$$
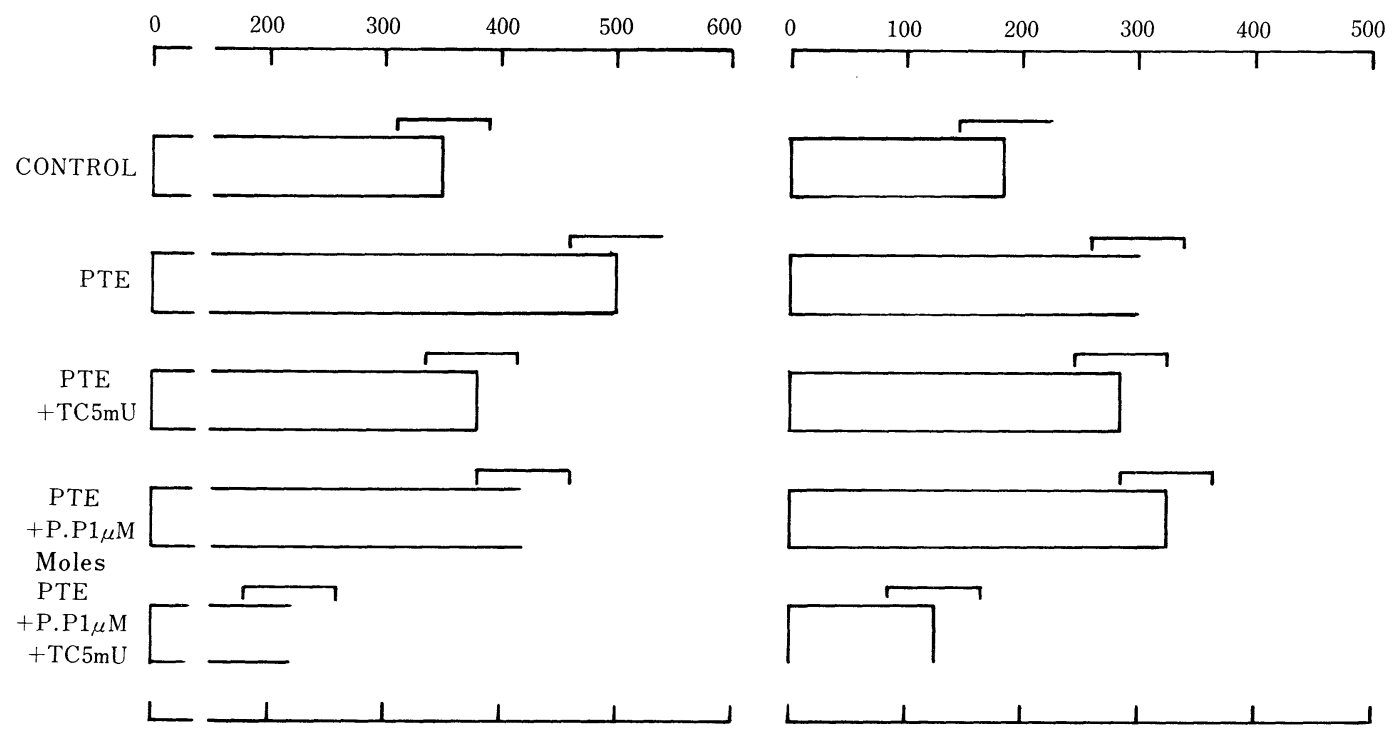

Fig. 6. Pyrophosphate enhancement of the inhibitory effect of TC on the release of Ca45 in PTE stimulated cultures. Each point represents the mean of 5 cultures. 


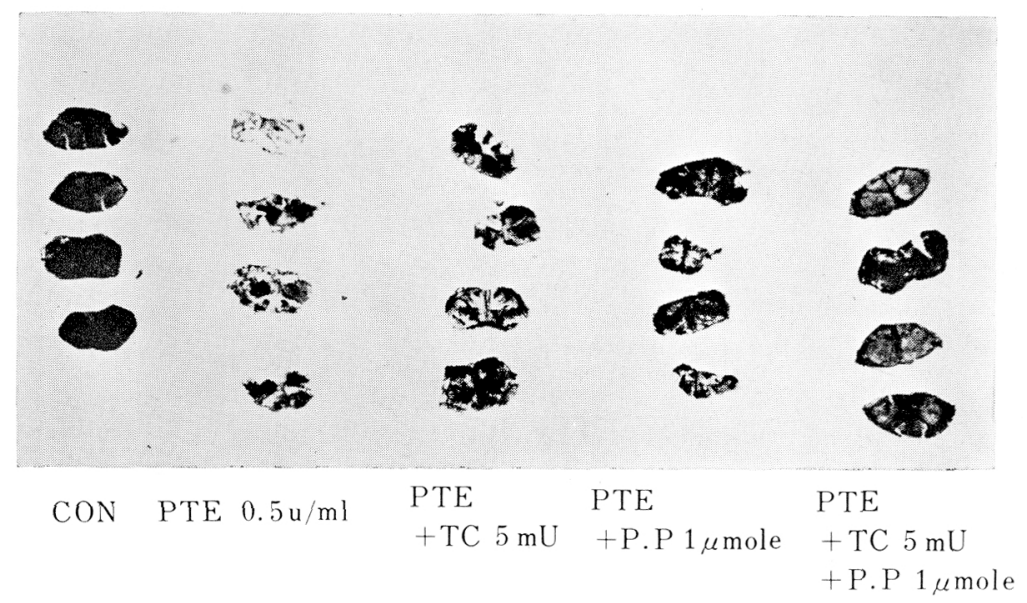

Fig. 7 Pyrophosphate enhancement of TC inhibition of bone resorption induced by PTE in a 6 day culture.

regarded as a synergistic rather than an additive effect. After 4 days of culture, no significant inhibition of $\mathrm{Ca}_{45}$ release occurred in the presence of TC. This was consistent with the previous result. However, addition of pyrophosphate apparently prevented the loss of the TC effect. Addition of pyrophosphate thus appears to restore the reactivity of the bone to TC apparently lost after 4 days culture. Gross appearance of the calvariae stained by Von Kossa method after 6 day culture also indicated the pyrophosphate enhancement of TC on PTE induced bone resorption. (Fig. 7)

\section{Discussion}

Although the bone culture system developed by Goldhaber contributed a great deal to the elucidation of the mechanism of bone resorption (Goldhaber, 1965), one of the disadvantage of this technique might be the difficulty in the evaluation of bone resorption quantitatively, especially at the early stage of the culture.

In an attempt to express the bone resorption more quantitatively, a modification was made to this system by measuring the release of previously incorporated $\mathrm{Ca} 45$ from the calvariae into the medium according to the method of Raisz (1965). The combination of the two parameters, gross appearance of the calvariae stained by the Von Kossa method and the release of previously incorporated $\mathrm{Ca}_{45}$ in the medium enabled us to show the small difference in the bone resorption much more quantitatively.

Considerable evidence for the inhibitory effect of TC on bone resorption has already been presented through studies in vivo (Milhaud, 1968; Johnston, 1966; Wallach, 1967; Martin, 1966; O'riordan, 1966) and in vitro (Friedman et al., 1965; Aliapoulios et al., 1966; Gaillard, 1966; Reynolds et al., 1968).

It is also known that TC can block both the early effect of PTH on calcium transport and the subsequent cell transformation in tissue culture. (Raisz et al., 1967) However, TC does not appear to act simply as a competitive antagonist to PTH since its initial effect can not be overcome by increasing doses of PTH. 
Moreover, $\mathrm{TC}$ inhibits resorption from either vitamin A stimulated or unstimulated bones in the absence of PTH. (Friedman et al., 1968)

Recently, Raisz et al. (1967) made an interesting observation that during the prolonged culture the inhibitory effect of TC on PTE stimulated bone resorption were only temporary despite repeated administration of fresh TC.

They named this phenomenon "escape" and claimed that it required the continuous presence of TC preparation in the culture medium. Our finding that $\mathrm{TC}$ is only effective during the first 2 days and not for the subsequent 2 days in the culture under constant stimulation with PTH is consistent with their finding. However, the fact that bone is not completely insensitive to the inhibitory effect of large doses of TC even after prolonged culture might suggested that this escape phenomenon merely represents a shift in dose-response curve as seen in acquired drug tolerance.

There is a possibility that our finding may be different from the escape phenomenon originally described by Raisz, since we have used the heterogenous porcine TC in our system (mouse calvariae) and Raisz et al. used the homogenous rat TC in their system (rat bone).

Raisz et al. (1967) claim that the escape phenomenon can also occur in vivo. However, an unpublished experiment (Orimo, 1969) showed no indication of reduced effects of TC after repeated injections. In rats treated with porcine TC twice daily for 3 weeks, TC was as effective in producing hypocalcemia in the first week as at the end of the period. If this escape phenomenon can also occur in human, it will be a serious problem in its therapeutic trial in disorders of calcium metabolism. Administration of phosphate has been shown to lower blood calcium probably through the increase in bone mineralization. (Pechet et al., 1967) Pyrophosphate also shares such a hypocalcemic property according to the results of the previous study (Orimo et al., 1969). Fleisch et al., (1962) are of the opinion that pyrophosphate, a normal constituent of plasma, may be important in inhibiting calcification both in vitro and in vivo. When apatite crystals are equilibrated with a solution in which the final concentration of pyrophosphate is that of plasma, pyrophosphate has two important effects on their behavior: prevention of the further growth of the crystals (Fleisch et al., 1966a) and the retardation of their rate of dissolution (Fleisch et al., 1966b). Our findings that the addition of pyrophosphate markedly inhibited the release of previously incorporated $\mathrm{Ca}^{45}$ into the medium, together with the histological evidence, strongly suggest the pyrophosphate inhibition of bone resorption. It has been suggested that mineralization will be initiated by removal of the inhibitor, pyrophosphate, through the action of pyrophosphatase. The fact that the amount of pyrophosphate sufficient to inhibit soft tissue calcification apparently has no histological effect on the further calcification and growth of already calcified bone in intact rat (Irving et al., 1966) might be due to the large amount of pyrophosphatase in the bone. Particularly interesting is the enhancement of pyrophosphate on the response of bone to TC in our system. Since the addition of pyrophosphate alone markedly inhibits the PTE induced bone resorption, the mechanism of the pyrophosphate enhancement of TC effect may be due to further inhibition of bone resorption by pyrophosphate and TC.

Furthermore, our results suggest that pyrophosphate appears to inhibit the occurrence of escape phenomenon with TC.

According to our unpublished data (Orimo et al., 1969), neutral phosphate also enhances the TC effect in this system. Addition of 3 $\mu$ moles of neutral phosphate which is ineffective alone, markedly enhanced the inhibitory effect of $5 \mathrm{mU}$ of TC on the PTE induced bone resorption. Furthermore, the addition of neutral phosphate apparently inhibited the occurrence of escape phenomenon with TC. This enhancing effect of phosphate on the response to TC was originally found in rat by Hirsch (1968). Our present in vitro data are consistent 
with these in vivo findings and clearly shows the enhancing effect of phosphate and pyrophosphate at the bone level.

\section{Acknowledgment}

The authors are indebted to Mr. K. Ohnishi and other staff of the Sankyo Central Research Laboratories for their cooperation. TC was kindly supplied by Eli Lilly and Co. Technical assistance of Y. Yonekubo is appreciated.

\section{Reference}

Aliapoulios, M. A., P. Goldhaber and P. L. Munson (1966). Science 151, 330.

Fleisch, H., R. G. G. Russell and F. Straumann (1966). Nature 212, 901.

Fleisch, H., J. Maerki and R. G. G. Russell (1966). Proc. Soc. Exp. Biol. Med. 122, 317.

Friedman, J. and L. G. Raisz (1965). Science 150, 1465.

Friedman, J., W. J. W. Au and L. G. Raisz (1968). Endocrinology 82, 149.

Gaillard, P. J. Fourth Europ. Symp. on Calcified Tissues, Excerpta Medica Found. Int. Congress Series, No. 120, p. 32 (1966).

Goldhaber, P. The Parathyroid Glands, University Press, Chicago, p. 158 (1965).

Hirsch, P. F. Calcitonin, Proceedings of the
Symposium on Thyrocalcitonin and the $\mathrm{C}$ cells. Heinemann, London, p. 11 (1968).

Irving, J. T., D. Schibler and H. Fleisch. (1966). Proc. Soc. Exp. Biol. Med. 123, 33w.

Johnston, C. C., Jr., and W. P. Deiss, Jr. (1966). Endocrinology 78, 1139.

Martin, T. J., C. J. Robinson and I. Macintyre (1966). Lancer 1, 900.

Milhaud, G., A. M. Perault and M. S. Moukhtar (1968). Compt. Rend. Acad. s.c. (Paris) 261, 813.

Munson, P. L. and P. F. Hirsch (1966). Clin. Orthoped. 49, 209.

Orimo, H., T. Fujita and M. Yoshikawa. Endocrinol. Japon. (16, 309, 1969).

Orimo, H., L. L. Darago and P. F. Hirsch. Endocrinology (in press).

O'Riordan, J. L. H. and G. Aurbach (1966). Ibid. 78, 1139.

Pechet, M. M., E. Bodadilla, E. L. Carrol and R. H. Hesse (1967). Amer. J. Med. 43, 696.

Raisz, L. G. (1965). J. Clin. Invest. 44, 103.

Raisz, L. G., W. Y. W. Au, J. Friedman and I. Nieman (1967). Am. J. Med. 42, 684.

Raisz, L. G., J. S. Brand, Y. W. Williams and I. Niemann (1967). Abstract of the 3rd Parathyroid Symposium.

Reynolds, J. J. (1968). Proc. Roy. Soc. (London) Ser. B. 170, 61.

Snedecor, G. W., and W. G. Cochran Statistical Methods, 6th ed. Iowa State University Press, Iowa p. 271 (1967). 